\title{
Jets from neutron star X-ray binaries: towards a unified scheme
}

\author{
Simone Migliari \\ European Space Agency/European Space Astronomy Center, Apartado/P.O. Box 78, \\ Villanueva de la Cañada, E-28691 Madrid, Spain, email: smigliari@sciops.esa.int
}

\begin{abstract}
Systematic multi-wavelength studies of neutron stars (NSs) have shown a jet and disk-jet coupling phenomenology which resembles, although with some important differences, that observed in black holes; ultra-relativistic transient ejection, steady compact jets, accretionejection cycles are indeed observed in NSs. I will review our observational knowledge of jet in NS X-ray binaries, focusing on the role of the parameters of the system which might be involved in the production of jets. First, I will discuss the role of the accretion rate, presenting a unified scheme for accretion-jet production throughout the different sub-classes of low-magnetic field NSs. Then, I will attempt to (make the first steps to) quantify the role of spin and magnetic field in powering the jet.
\end{abstract}

Keywords. stars: neutron, X-rays: binaries, radio continuum: general, ISM: jets and outflows.

\section{Introduction}

Jets are spectacular and powerful phenomena observed in a number of astrophysical objects, from non-relativistic to relativistic systems, such as Young Stellar Objects (YSOs), cataclismic variables (CV), X-ray binaries (XRBs) and active galactic nuclei (AGN; and are supposed to be at the origin of the powerful gamma-ray bursts). In the search for the elements and parameters contributing to the formation of jets, key is the cross comparison of the different jet systems. The accretion disk is a common denominator for all these systems and seems to be essential to the formation of their jets. However, differences in the jet behaviour among these systems, indicate that also parameters proper of the accreting star might play a role in the jet production.

\subsection{The sub-calsses of neutron star X-ray binaries}

Low-magnetic field NS XRBs are commonly divided into two broad classes, based on their X-ray spectral and timing properties, and named after the shape of their colorcolor diagram (CD) and hardness-intensity diagram (HID): atolls and Z (Fig. 1, left). Atoll sources have luminosities between 0.01 and 0.5 Eddington, while $\mathrm{Z}$ sources are persistently above 0.5 Eddington and sometimes even at super-Eddington luminosities. In their CD track, atolls show 'branches' (X-ray states) where the soft disk emission dominates more or less the spectrum over a non-thermal high-energy emission: a hard branch called island state, and a soft branch called banana state. The $\mathrm{Z}$ track typically show three branches, from softer to harder: Flaring Branch (FB), Normal Branch (NB) and Horizontal Branch (HB). Based on the orientation and presence of these branches, both atolls and $\mathrm{Z}$ sources can be further divided into sub-classes. In the following, I will divide the NSs into five sub-classes: Atoll-Hard (mostly island), Atoll-Soft (mostly banana), Atoll-GX (persistently bright, mostly banana), Z-Sco (or Sco-like) and Z-Cyg (or Cyg-like). It is still a matter of debate if variations of the mass accretion rate alone can explain the variety of NS sub-classes and the different branches within a sub-class. 

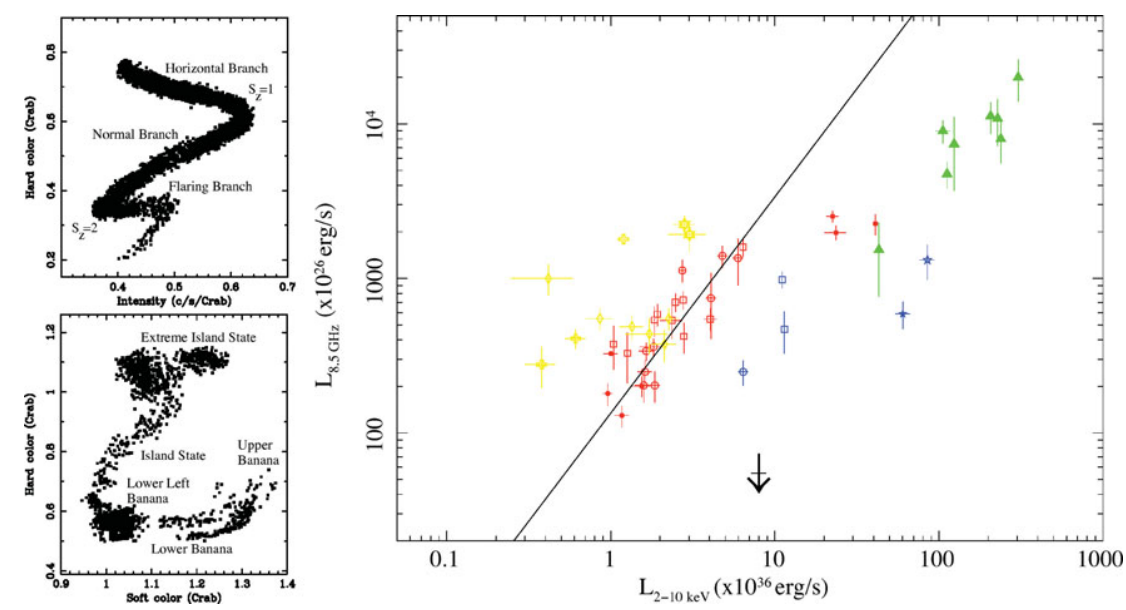

Figure 1. Left: typical CD of an Atoll and HID of a Z-type NS (van der Klis 2006). Right: Updated X-ray/radio luminosity plots of NSs: in red atolls in hard state and state transition; in blue atolls in soft state; in green Z sources; in yellow AMXPs. The solid line fits 4U 1728-34 with a power law with $\Gamma=1.4$; The arrow shows the radio $3 \sigma$ upper limit of GX $9+9$, showing the quenching at $\sim 10 \%$ Eddington.

\subsection{XTE J1701-462: the Rosetta Stone}

XTE J1701-462 is the first source showing the transition between Z-types and atoll-type, during an X-ray outburst, going through all the sub-classes and branches withing a few months (Homan et al. 2010 and references therein). Homan et al. (2010) showed that the average mass accretion rate (using the $2-2.9 \mathrm{keV}$ count rate as a proxy) seems to be responsable for the different sub-classes, i.e. in order of increasing accretion rate: atoll-hard, atoll-soft, atoll-GX, Z-Sco and Z-Cyg. Nevertheless, it seems that variations in the accretion rate can trace the evolution of the CD thoughout all the NS sub-classes, the average mass accretion rate alone is not able to account for variations between the different branches within a sub-class (especially in Z sources), and at least an additional parameter seems to be needed.

\section{The sample}

The sample presented here consists of all the NS XRBs with i) a detected radio counterpart, ii) a quasi-simultaneous X-ray/radio coverage and iii) an estimated distance (e.g. Nelemans \& Jonker 2004 for uncertainties). I report here only the radio detections (with the exception of GX9+9; see below). For the radio characteristics of most of the sources in the sample we refer to Migliari \& Fender (2006) and references therein. In the following sections, I report new sources, updates and notes on key observations.

\subsection{Radio emission from Atolls}

$4 U$ 0614+091: The four radio detections of the source are reported in Migliariet al.(2010). The source was steady in its hard state and emission is consistent with being opticallythick synchrotron coming from a (slightly variable) compact jet. 

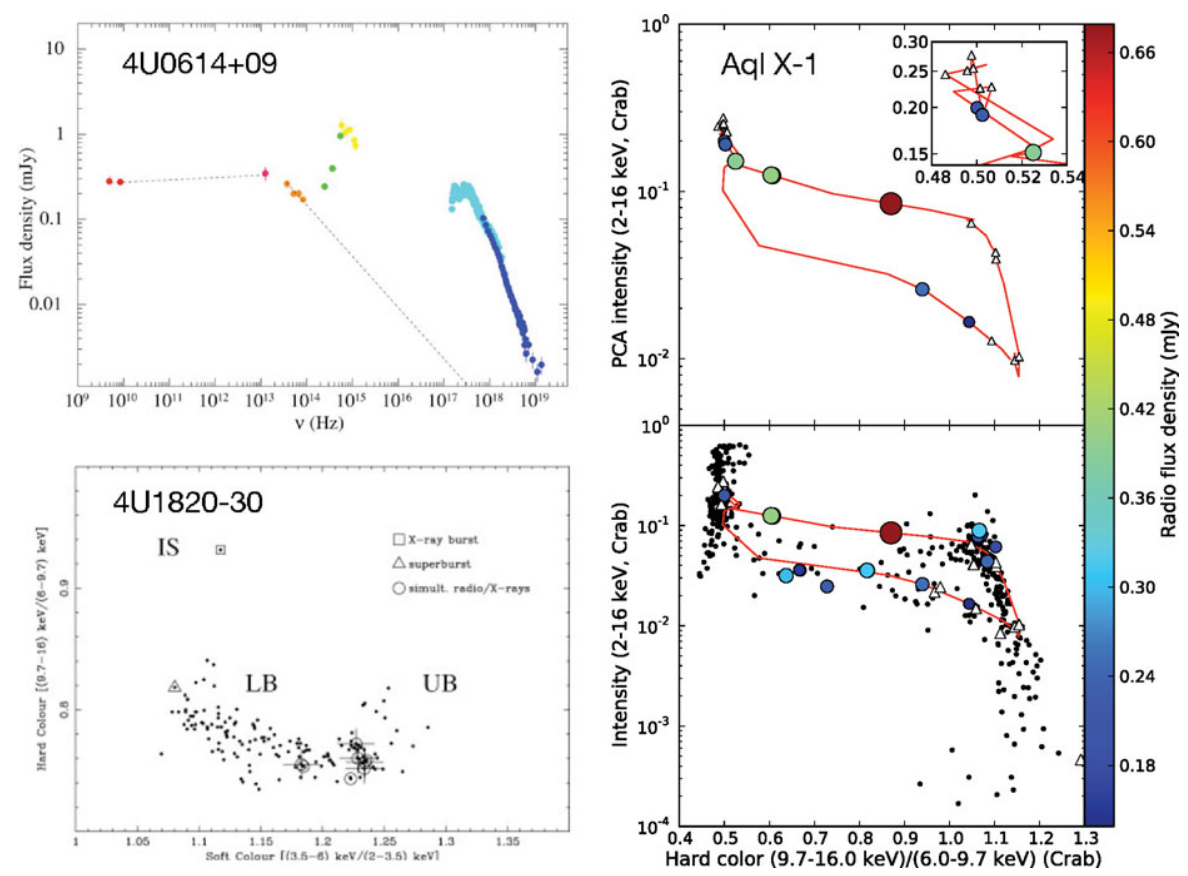

Figure 2. Radio detections of atolls. Upper left: broadband spectrum of $4 \mathrm{U} 0614+101$ when steadily in its hard state (Migliari et al. 2010). Lower left: CD of 4U 1820-30 showing with circles the radio detection when steadily in its soft state (Migliari et al. 2004). Right: HID of AqlX-1 showing the evolution of the radio emission during X-ray outbursts. The top panel shows the 2009 outburst, while the lower panel all the available observations (Miller-Jones et al. 2010).

Aql X-1: Miller-Jones et al. (2010) report on daily VLA and VLBA observations of Aql X-1 during the 2009 outburst, the best radio coverage of a NS XRB outburst to datef. The source has been detected 14 times in the radio band (see also Tudose et al. 2009). The strongest radio flux density is observed during the X-ray state transition. Also a radio quenching during the soft state and after the state transition is observed, similar to that found in BHs. The radio emission is always optically-thick along the whole X-ray outburst, indicating that it arises from a compact jet.

$4 U$ 1820-30 and Ser X-1: Migliari et al. (2004) reported radio emission when they were steady in their soft state (banana). The radio luminosity is comparable to that observed in the hard state from other sources. This is different from what has been observed so far in BHs, whose radio emission is at least an order of magnitude lower than that in hard state. The radio spectrum was consistent with being optically thick, indicating a compact jet.

MXB 1730-335 (the Rapid Burster): The source has been detected in its soft state, near the peak of two X-ray outbursts (Rutledge et al. 1998; Moore et al. 2000). Contrary to what is expected from $\mathrm{BHs}$, the radio spectra of the two dual-band observations are consistent with a compact jet.

Quenching: in atolls, radio emission is observed to increase with X-ray luminosity and then quench at a few to ten per cent the Eddington limit: Aql X-1 (Miller-Jones et al.

$\dagger$ JACPOT collaboration: http:www.astro.virginia.edu/xrb_jets 
2010), possibly 4U 1728-34 (Migliari et al. 2003), and atoll-GX as GX 3+1 (Berendsen et al. 2000) and GX 9+9 (see Fig. 1; in prep.).

\subsection{Radio emission from Z-type neutron stars}

Eight† highly-accreting Z-type XRBs are known: six persistent (Sco X-1, GX 17+2, GX 349+2, Cyg X-2, GX 5-1, GX 340+0), one transiting from atoll to Z type (XTE J1701462) and the peculiar Cir X-1: all of them have been detected in the radio band. The radio emission in $\mathrm{Z}$ souces in extremely variable and it seems to be related to the $\mathrm{X}$-ray state. Strictly simultaneous radio/X-ray studies have been reported for six Z sources: GX 17+2 (Penninx et al. 1988; Migliari et al. 2007), Cyg X-2 (Hjellming et al. 1990a; Migliari, Miller-Jones et al. in prep.), Sco X-1 (Hjellming et al. 1990b; Fomalont et al. 2001; Bradshaw et al. 2003), GX 5-1 (Tan et al. 1992), 4U 1701-462 (Fender et al. 2007) and Cir X-1 (Soleri et al. 2009).

Z-Sco: Cyg X-2, Sco X-1 and GX 17+2: Simultaneous X-ray radio observations show a link between the $\mathrm{X}$-ray branch and the intensity of the radio emission for the three sources. Within each source, the radio flux density increases from the HB to the NB and it is weakest in the FB (see a sketch inset in Fig. 3). Radio brightenings have been observed during state transitions between HB and NB (Sco X-1) and between FB to NB $($ GX $17+2)$. Both optically-thick and optically-thin radio spectra have been observed. Therefore, $\mathrm{Z}$ sources, contrary to atolls, can launch transient jets.

Z-Cyg: Cyg X-2 and GX 5-1: The radio coverage for these two sources is sparse and only part of the HID track is observed simultaneously with X-rays. GX 5-1 shows radio emission in the $\mathrm{HB}$ and an enhanced flux density when the source transits to the NB (Tan et al. 1992). The increase in radio emission is possibly associated to the HB-NB transition. Cyg X-2 shows stronger radio emission at the FB-NB apex, while the jet seems to be quenched when it is steadily in the NB (in prep.). No dual-band observations are available to investigate the nature of the jet in Cyg X-2. The radio spectra in GX 5-1 show both optically thick and optically thin synchrotron emission.

\section{A Unified Scheme: jet power vs accretion rate}

In Fig. 3, I present with a unified scheme, the puzzled radio behaviour of low-magnetic field NSs observed up to now throughout the different sub-classes, i.e. as a function of the mass accretion rate. The results can be summarized as follows.

- Radio emission has been detected from both atolls and Z sources.

- On average, the radio flux density detected increases with accretion rate, from atolls to $\mathrm{Z}$ sources.

- Radio emission has been detected in all branches of atolls and Z sources.

- Atolls show only optically-thick synchrotron radio emission, consistent with a compact jet, even during X-ray state transitions and outbursts.

- At a few per cent of the Eddington limit, the compact jet radio emission seems to be quenched. (The lowest radio upper limit comes from the 'atoll-GX' GX 9+9 at approximately 10\% Eddington.)

- Z sources show optically-thick and optically-thin synchrotron radio emission, evidence for the presence of a powerful compact jet and transient jets.

- In both atolls and Z sources, there is evidence for an association between X-ray state (branch)transitions and enhanced jet activity.

$\dagger$ Nine if we also count GX $13+1$, which is an hybrid atoll-Z source 


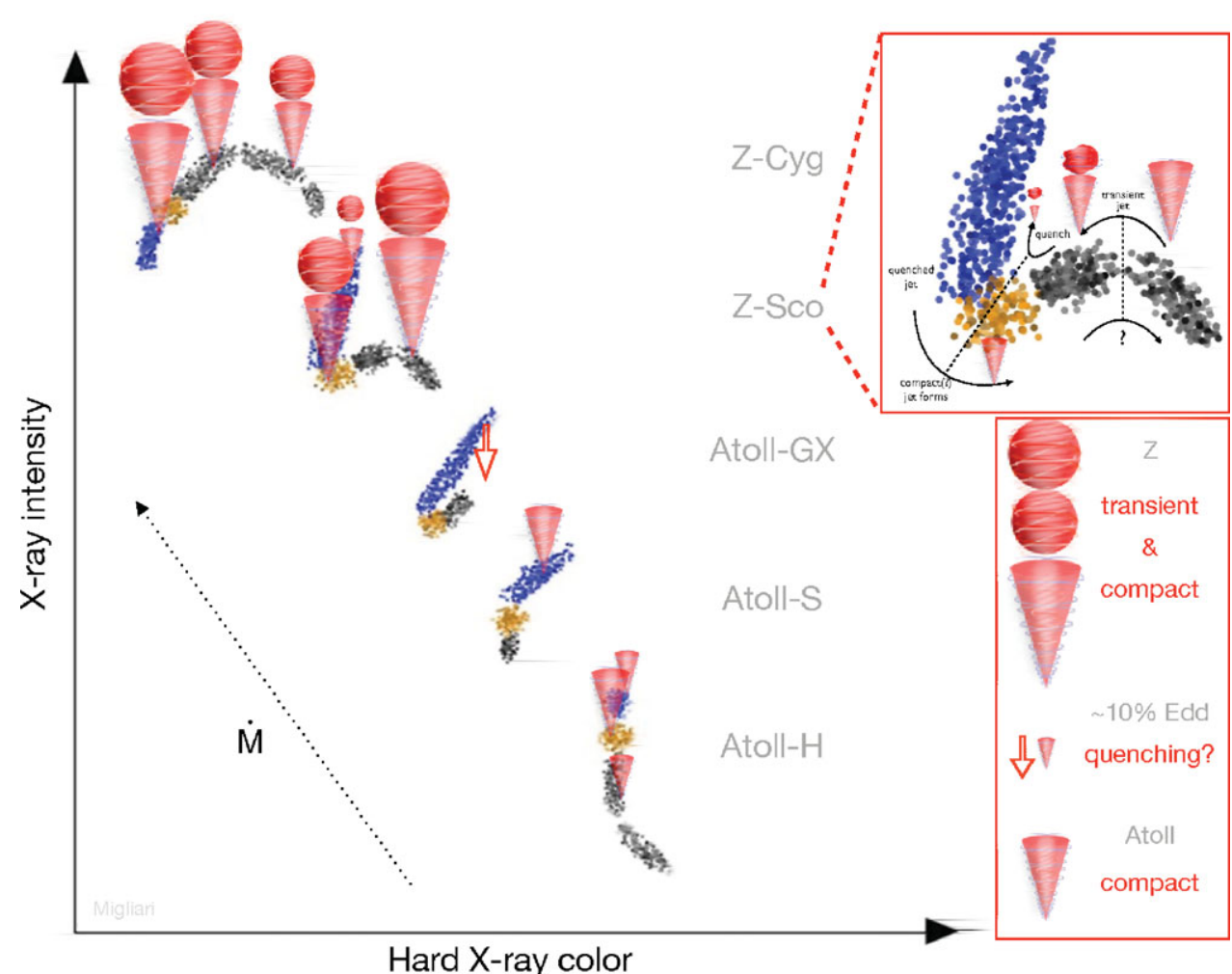

Figure 3. A unified scheme for low-magnetic field NSs: sub-classes and radio emission as a function of the accretion rate. We show the typical HID track of five main sub-classes. In blue we represent the FB and the banana states and in orange the island-banana apex which evolves into the FB-NB apex. The red cones represent the observed compact jet emission: the brighter the jet, the bigger the cone. Transient jets are shown as detached blobs.

\section{Neutron star magnetic field vs jet power}

Contrary to BHs, NSs in XRB systems show the presence of strong surface magnetic fields. The magnetic field can be directly measured only by observing cyclotron emission lines in the X-ray spectrum. Cyclotron lines have been observed in $15 \mathrm{X}$-ray binary pulsars, giving a magnetic field near the poles in the range $10^{11}-10^{13} \mathrm{G}$.

\subsection{Radio upper limits from high-magnetic field neutrons}

We made a systematic analysis of radio observations of high-magnetic field neutron star X-ray binaries with estimated stellar magnetic field and known distance (Fig. 4, right; Migliari, Miller-Jones et al., in prep.). The figure includes new radio observations of Her X-1, 4U 0115+634, 4U 1907+09 and A 0535+26, which give the most stringent radio upper limits of the class to date. Only one high-magnetic field NS have been detected in the radio band (4U 1223-624; Pestalozzi et al. 2009). However, the radio flux density is consistent with emission from the wind of the companion star which should dominate the radio spectrum. Furthermore, the reported variability between orbital phases which Pestalozzi et al. (2009) speculate it is coming from a transient jet, show large uncertainties, i.e. less than $3 \sigma$ significance, and therefore must be confirmed. Tudose et al. (2010) reported the only constraining X-ray radio upper limits to date for A $0535+26$ during the X-ray outburst of July-August 2010: our WSRT observations show upper limits an 
order of magnitude below the radio luminosity of atolls in hard state at the same X-ray luminosity (at $\left.\sim 10^{36} \mathrm{erg} / \mathrm{s}\right)$.

Does a high-magnetic field quench the jet production? The picture is still blurred. No detections of high-magnetic field X-ray pulsars have been observed so far. However, if we take into account also the soft X-ray luminosity, the radio upper limits are still consistent with what expected in low-magnetic field NSs (X-ray/radio luminosity correlation). Only one observation is off, i.e. A $0535+26$. Therefore, although hints that a high magnetic field may quench the jet production are growing, further investigation is needed before drawing any reliable conclusion.

\section{Spin vs jet power}

In NSs, the spin might affect the jet production through the interaction of the accretion disk and the magnetic field anchored to the NS poles.

\subsection{Measuring the spin of the neutron star}

Unlike in BHs, the spin in NS XRBs can be directly observed by non-thermal emitting particles accelerated by the polar magnetic field of the rotating NS. This is the case for high-magnetic field XRB pulsars and accretins millisec X-ray pulsars (AMXPs), where a coherent pulsation is directly detected. In low-magnetic field NS XRBs, the spin period can be observed indirectly as 'burst oscillation', i.e. quasi-coherent oscillations during type-I X-ray bursts. In the three sources where both direct pulsation and burst-oscillation have been observed, the burst oscillation was consistent with the spin frequency $\nu_{s}$ (Chakrabarty et al. 2003; Strohmayer et al. 2003; Altamirano et al. 2008). Twin $\mathrm{kHz}$ quasi periodic oscillations (QPOs) in the X-ray power spectra have been observed for a number of NS XRBs. The difference between the peak frequencies $\nu_{k H z}$ of the two QPOs seems to be in some relation with the spin frequency (either consistent with or half $\nu_{s}$ deending on the source; e.g. van der Klis 2006). Below, for those sources where a direct measurement of the pulsation of the NS is not observed, we choose to equal the spin to the average burst oscillation frequency or the highest $\Delta \nu_{k H z}$ measured.

\subsection{Estimating the jet power}

Following Fender et al. (2010), I estimated the (ranking) jet power of the sample, using as a proxy the normalization of the power law fitting the sources in the X-ray/radio luminosity plane (Fig. 1, right). Only two atoll sources, 4U 1728-34 and Aql X-1, span an order of magnitude in X-ray luminosity and show a correlation in the radio/X-ray luminosity plane. The slope is different for the two sources: $\Gamma \sim 1.4$ and $\Gamma \sim 0.6$, respectively for 4U 1728-34 and Aql X-1 (Migliari et al. 2003; Tudose et al. 2009; MillerJones et al. 2010). I chose to treat the average NS radio emission as dominated by a compact jet. I fitted each source in the X-ray/radio luminosity plane with a power law, fixing the slope first to 1.4 and then to 0.6 , and plot both the normalizations against the spin frequencies.

\subsection{Spin-jet power correlation?}

The rank in jet power $\mathrm{L}_{J e t}$ among the NSs, estimated as the rank in the normalization of the law $\mathrm{L}_{R} \propto \mathrm{L}_{X}^{1.4}$ (i.e. fit to $4 \mathrm{U}$ 1728-34 and expected theoretically for radiatively efficient accretion when $\dot{M} \propto L_{J e t}$; see also Körding et al. 2007), show an apparent correlation to the spin: the non-parametric Spearman rank statistics gives a probability of 3e-2 against the null hypothesis of an uncorrelated data set (Fig. 4). However, given the outlier ( $4 \mathrm{U}$ 0929-314 with $\nu_{s}=185 \mathrm{~Hz}$ ) and the uncertainties on the measurements 

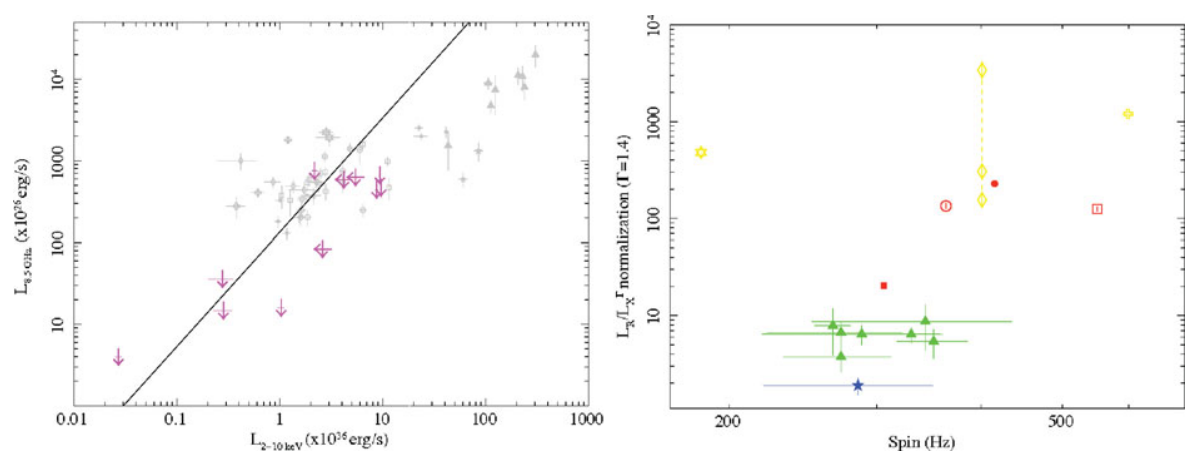

Figure 4. Left: Upper limits of high magnetic field X-ray pulsars (purple) superimposed to the other NSs (grey). Right: Spin vs X-ray/radio luminosity Normalization of a fitting power law with $\Gamma=1.4$.

discussed above, a much better statistics is necessary to confirm the relation. If we plot the spin frequency against the normalization fixing the law $\mathrm{L}_{R} \propto \mathrm{L}_{\dot{X}}^{0.6}$ (fit to Aql $\mathrm{X}-1$ ), there is no obvious correlation.

A lack of evidence of a relation between spin and jet power, suggests that the main driver of wind/jet is not strictly linked to the accreting object. This would point towards the extended-disk model as the most likely mechanism (Blandford \& Payne 1982). The same conclusion for YSOs has been derived by Anderson et al. (2003) and Ferreira et al. (2006), measuring, at least for the outer portion of the jet, a large launching disk radius for a sample of T-Tauri stars. They also discuss the existence of a coaxial, faster flow inside the outer jet, possibly powered by one of the other two mechanisms (X-wind or stellar-wind). Note that also in NS XRBs, a faster, relativistic jet superimposed to a slower radio ejection has been observed in two NS systems (Sco X-1 and Cir X-1; Fomalont et al. 2001; Fender et al. 2004) and possibly also in SS 433 (where the nature of compact object is still unknown; Migliari et al. 2005). In these cases, the jet may tap power from the angular momentum of compact object itself, and the spin and stellar magnetic field may in fact be crucial elements.

\section{Models for jet production in NS XRBs, what are we looking for?}

Based on the results described in this work, I herewith list some results that I think a model for jet formation in NSs should address:

- when liberated, the average jet power correlates with the average accretion rate.

- within a sub-class, the observed jet power depends on (at least) a second parameter, which regulate the channels through which the power is liberated (jet, wind,...) and is related to X-ray state transitions (e.g. instantaneous accretion rate at a certain radius? disk instabilities?).

- transient, optically-thin synchrotron radio emission is observed only in Z sources, i.e. at a high accretion rate, above a few tens per cent of the Eddington luminosity limit.

- faster, underlying jet emission, superimposed to a slower jet, is observed in at least two highly-accreting NS sources. Is there a two-modes jet production? are two processes for jet formation coexisting as suggested for YSOs?

- if the process of transient/faster jet is powerded by the spin in both NSs and BHs, then the NS must find a way to produce a jet which is comparable or even faster than 
that observed in stellar BHs, although with a spin of $a<0.2$, which is likely less than that in the BHs we observe.

- There is no quantitative proof yet that either the spin or the magnetic field play a significant role in powering the jet. Nor there is that they do not play a role. However, if we take the results with caution, we might say that there are hints. The indication that a high magnetic field may quench the jet production is growing, since, despite the (still ongoing) trials, there is still no evidence for radio emission from high-magnetic field X-ray pulsars (yes, lack of evidence is not evidence of lack, but...); 2) there is a possibility (very weak, not believable yet considering the uncertainties) that to a higher spin corresponds a stronger jet, if the X-ray/radio luminosity correlation for a compact jet has $\Gamma \sim 1.4$.

\section{Acknowledgements}

I am thankful to James Miller-Jones for the many discussions and for comments on a draft of this work.

\section{References}

Anderson, J. M., Li, Z.-Y., Krasnopolsky, R., \& Blandford, R. D. 2003, ApJL, 590, L107

Blandford, R. D., \& Payne, D. G. 1982, MNRAS, 199, 883

Bradshaw, C. F., Geldzahler, B. J., \& Fomalont, E. B. 2003, ApJ, 592, 486

Chakrabarty, D.et al. 2003, Nature, 424, 42

Fender, R. et al. 2004, Nature, 427, 222

Fender, R. et al. 2007, MNRAS, 380, L25

Fender, R. P., Gallo, E., \& Russell, D. 2010, MNRAS, 406, 1425

Fender, R. 2010, Lecture Notes in Physics, Berlin Springer Verlag, 794, 115

Ferreira, J., Dougados, C., \& Cabrit, S. 2006, A\&A, 453, 785

Fomalont, E. B., Geldzahler, B. J., \& Bradshaw, C. F. 2001, ApJ, 558, 283

Hjellming, R. M., Han, X. H., Cordova, F. A., \& Hasinger, G. 1990, A\& A, 235, 147

Hjellming, R. M., et al. 1990, ApJ, 365, 681

Homan, J., et al. 2010, ApJ, 719, 201

Körding, E. G. et al. 2006, MNRAS, 369, 1451

Migliari, S., \& Fender, R. P. 2006, MNRAS, 366, 79

Migliari, S. et al. 2003, MNRAS, 342, L67

Migliari, S. et al. 2004, MNRAS, 351, 186

Migliari, S., et al. 2007, ApJ, 671, 706

Migliari, S., et al. 2010, ApJ, 710, 117

Miller-Jones, J. C. A., et al. 2010, ApJL, 716, L109

Penninx, W. et al. 1988, Nature, 336, 146

Pestalozzi, M. et al. 2009, A\&GA, 506, L21

Psaltis, D., \& Chakrabarty, D. 1999, ApJ, 521, 332

Rutledge, R., Moore, C., Fox, D., \& Lewin, W. 1998, ATel, 8, 1

Shu, F., Najita, J., Ostriker, E., Wilkin, F., Ruden, S., \& Lizano, S. 1994, ApJ, 429, 781

Soleri, P., Tudose, V., Fender, R., van der Klis, M., \& Jonker, P. G. 2009, MNRAS, 399, 453

Tan, J. et al. 1992, ApJ, 385, 314

Tudose, V., Migliari, S., Miller-Jones, J. C. A. et al. 2010, ATel, 2798, 1

Tudose, V., Fender, R. P., Linares, M., Maitra, D., \& van der Klis, M. 2009, MNRAS, 400, 2111 van der Klis, M. 2006, Compact stellar X-ray sources, 39 


\section{Discussion}

DAVID MEIER: Marina Romanova has simulated the magnetic propeller mechanism which produces jets from the magnetic neutron star's rotation. This too gives a jet power $P_{J} \propto \dot{M}^{\beta}$, where $\beta \sim 1$. So, just because $P_{J} \propto \dot{M}$ does not mean that the jet cannot be pulsar driven.

Migliari: Absolutely correct. As a note, I must add that the slope of the X-ray/radio luminosity correlation from which a $P_{J} \propto \dot{M}$ can be inferred assuming a radiatively efficient disk emission (i.e. $L_{X} \propto \dot{M}$ ), is not certain yet. One source, 4U1728-34 shows a relation $L_{R} \propto L_{X}^{\Gamma}$ with $\Gamma \sim 1.4$, while Aql X-1 shows $\Gamma \sim 0.6$.

Elisabete M. De Gouveia dal Pino: My question is also related to Romanova and collaborators' propeller models for magneto-rotational launching. You said that you looked for very high $\left(B>10^{12} \mathrm{G}\right) \mathrm{NSs}$ in order to see a possible evidence for a jet quenching, but actually normal NSs can have even weaker magnetic fields and B can actually help launching. Why is that so? were you looking for magnetar-like NSs?

MigLIARI: When we started searching systematically for a possible radio synchrotron emission from high-magnetic field X-ray pulsars, we were looking for any possible effect of the magnetic field on jet emission, either quenching or active (as in the case of the propeller effect). We did not find any convincing quantitative proof for either the effect, yet. The indication for a quenching effect comes from the fact that you just mentioned: jets have been detected in weak magnetic field NSs, but not in high magnetic field NSs. However, again, there is no strong quantitative proof for a quenching effect, if we take into account the X-ray luminosity of the observed sources. We did not look for magnetars yet, which are even more extreme in magnetic field strength.

FEnG YUAN: Is the transient jet detected in AqlX-1 associated with X-ray state transition?

MigliARI: yes, with transitions from hard to soft and from soft to hard states. In both directions.

Christian Fendt: What is the role of the stellar magnetic field inclination? if the inclination is too high, jet formation will be disturbed/suppressed. Jet formation requires a high degree of axisymmetry.

MigliARI: Yes, I agree. Unfortunately, in our sample of high-magnetic field NSs we do not have information on the magnetic axis inclination, except for some possible constrain/selection bias coming from the fact that we selected the sources where we observe pulsations and cyclotron lines (the only way to measure directly the magnetic field), both coming from regions near the magnetic poles. 